\title{
ON THE LOJASIEWICZ EXPONENT OF THE GRADIENT OF A HOLOMORPHIC FUNCTION
}

\author{
ANDRZEJ LENARCIK \\ Kielce University of Technology \\ Al. Tysiaclecia Państwa Polskiego 7, 25-314 Kielce, Poland \\ E-mail: lenarcik@sabat.tu.kielce.pl
}

Finding out what is acceptable to the Lord. $($ Eph 5,10)

\begin{abstract}
The Łojasiewicz exponent of the gradient of a convergent power series $h(X, Y)$ with complex coefficients is the greatest lower bound of the set of $\lambda>0$ such that the inequality $|\operatorname{grad} h(x, y)| \geq c|(x, y)|^{\lambda}$ holds near $0 \in \mathbf{C}^{2}$ for a certain $c>0$. In the paper, we give an estimate of the Eojasiewicz exponent of grad $h$ using information from the Newton diagram of $h$. We obtain the exact value of the exponent for non-degenerate series.
\end{abstract}

1. Introduction. The main goal of this paper is to compute the Lojasiewicz exponent of the plane curve singularity using the Newton Polygon. Our theorem is the counterpart of the Kouchnirenko theorem [Kou] on the Milnor number in the case of two variables. We were inspired by the articles of Lichtin $[\mathrm{Li}]$ and Fukui $[\mathrm{Fu}]$ who proved some estimates for the Łojasiewicz exponent. However, our considerations are based on other ideas and enable us to calculate the Łojasiewicz exponent for non-degenerate power series. The organization of the paper is as follows. In Section 2 we present the main theorem with illustrative examples. Then we collect in Section 3 basic notions connected with the Newton diagrams. Our main reference is [BK]. A theorem concerning the Łojasiewicz exponent of a holomorphic mapping, determined by the pair of convergent power series, is given in Section 4. The theorem is helpful in a proof of the main result given in Section 6 . The proof is preceded by the theorems which describe connections between the Newton diagram of the series $h$ and the diagrams of its derivatives $\frac{\partial h}{\partial X}, \frac{\partial h}{\partial Y}$ (Section 5). These results are of independent interest.

We denote by $\mathbf{C}\{X, Y\}$ the ring of convergent power series with complex coefficients in variables $X, Y$. For every $h \in \mathbf{C}\{X, Y\}$ we use ord $h$, or respectively in $h$ to denote order or respectively initial form of the series $h$. If $z=(x, y) \in \mathbf{C}^{2}$, then $|z|=\max \{|x|,|y|\}$. We use conventions: $\inf \emptyset=+\infty$ and $\sup \emptyset=-\infty$.

Research supported by KBN grant 2 PO3 A 07908.

1991 Mathematics Subject Classification: Primary 14B05; Secondary 32S05.

Received by the editors: January 5, 1997.

The paper is in final form and no version of it will be published elsewhere. 
2. The main theorem. In this section the main result of the paper (Theorem 2.1) is formulated. The proof of the main theorem is given in Section 6 .

Any pair of convergent power series $f, g \in \mathbf{C}\{X, Y\}$ without constant term determines the germ $H=(f, g):\left(\mathbf{C}^{2}, 0\right) \longrightarrow\left(\mathbf{C}^{2}, 0\right)$ of a holomorphic mapping. An important local characteristic of $H$, developed by many authors [LJ-T], [KL], [Pł1], [ChK1] is the Łojasiewicz exponent

$$
l_{0}(H)=\inf \left\{\lambda>0: \max \{|f(x, y)|,|g(x, y)|\} \geq \text { const. } \cdot \max \{|x|,|y|\}^{\lambda} \text { near zero }\right\} .
$$

It is well known that $l_{0}(H)<+\infty$ if and only if the system of equations $f(X, Y)=$ $g(X, Y)=0$ has an isolated solution at $0 \in \mathbf{C}^{2}$. Let $h \in \mathbf{C}\{X, Y\}$ be a series with a singularity at $0 \in \mathbf{C}^{2}$, i.e. such that

$$
h(0,0)=\frac{\partial h}{\partial X}(0,0)=\frac{\partial h}{\partial Y}(0,0)=0 .
$$

We have $l_{0}(\operatorname{grad} h)<+\infty$ if and only if the curve $h=0$ has an isolated singularity at $0 \in \mathbf{C}^{2}$. Lu and Chang [LCh] (developing the results of Kuo [K], Kuiper [Kuip], Bochnak and Eojasiewicz $[\mathrm{BE}])$ proved that adding to the function $h$ monomials of order greater than $\left[l_{0}(\operatorname{grad} h)\right]+1$ does not change its topological type $\left({ }^{1}\right)$. The minimal integer with this property is called the $C^{0}$-sufficiency degree of $h$ and is denoted by $\nu_{h}$. Teissier $[\mathrm{Te}]$ showed that $\nu_{h}=\left[l_{0}(\operatorname{grad} h)\right]+1$. Kucharz $[$ Kuch1] found an example that the equality of this type is not true in the real case. Lichtin [Li] studied connections of $C^{0}$-sufficiency degree with the Newton diagram of series $h$.

For any power series $h(X, Y)=\sum h_{\alpha \beta} X^{\alpha} Y^{\beta}, h_{\alpha \beta} \in \mathbf{C}$, we put $\operatorname{supp} h=\{(\alpha, \beta)$ : $\left.h_{\alpha \beta} \neq 0\right\}$. The Newton diagram $\Delta_{h}$ of $h$ is the convex hull of $\operatorname{supp} h+\mathbf{R}_{+}^{2}$. The bound of the diagram $\Delta_{h}$ is the union of two half-lines and a finite number of compact and pairwise non-parallel segments. The Newton polygon $\mathcal{N}_{h}$ is the set of these segments. For any segment $S \in \mathcal{N}_{h}$ we define the initial form $\operatorname{in}(h, S)$ as the sum of monomials $h_{\alpha \beta} x^{\alpha} y^{\beta}$ over all $(\alpha, \beta) \in S$. We say that $h$ is non-degenerate on the segment $S \in \mathcal{N}_{h}$ if the system of equations $\frac{\partial}{\partial X} \operatorname{in}(h, S)=\frac{\partial}{\partial Y} \operatorname{in}(h, S)=0$ has no solutions in $(\mathbf{C} \backslash\{0\}) \times(\mathbf{C} \backslash\{0\})$. We say that $h$ is non-degenerate in Kouchnirenko's sense [Kou] if it is non-degenerate on all the segments from $\mathcal{N}_{h}$. For $S \in \mathcal{N}_{h}$, we denote by $\alpha(S)$ the abscissa of the point, where the line determined by $S$ intersects the horizontal axis, and by $\beta(S)$ the ordinate of the point, where the line intersects the vertical axis. For almost all series with the same Newton diagram Lichtin proved the following inequality

$$
\nu_{h} \leq\left[\max _{S \in \mathcal{N}_{h}}\{\alpha(S), \beta(S)\}\right]+1
$$

By "almost all" Lichtin meant a specific non-degeneracy condition stronger than the nondegeneracy in Kouchnirenko's sense. The result of Lichtin was improved by Fukui [Fu]. Fukui considered the case of $n$ variables. In the two-dimensional case, the result of Fukui can be written in the form

$$
l_{0}(\operatorname{grad} h) \leq \max _{S \in \mathcal{N}_{h}}\{\alpha(S), \beta(S)\}-1
$$

which gives

$$
\nu_{h} \leq\left[\max _{S \in \mathcal{N}_{h}}\{\alpha(S), \beta(S)\}\right]
$$

$\left({ }^{1}\right)[x]$ denotes the integer part of a real $x$. 
Fukui considered series with an isolated singularity at zero which are non-degenerate in Kouchnirenko's sense. Theorem 2.1, given below, is the essential improvement of the two results quoted above.
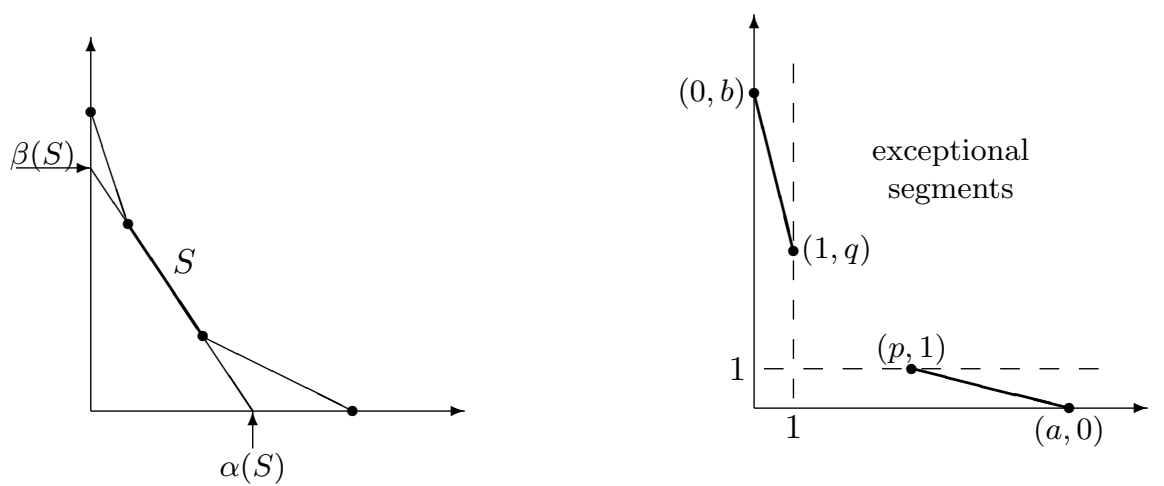

Any series $h \in \mathbf{C}\{X, Y\}$ will be referred to as nearly convenient if it is non-zero and the both distances between the diagram $\Delta_{h}$ and the axes are less than or equal to one. It is easy to show that any series $h$ with an isolated singularity is nearly convenient. The reduced Newton polygon $\mathcal{N}_{h}^{*}$ is obtained from the Newton polygon $\mathcal{N}_{h}$ by omitting the extreme segments in some situations. The segment nearest to the vertical axis is omitted when its lower end has the form $(1, q)$. The segment nearest to the horizontal axis is omitted when its upper end has the form $(p, 1)$. The segments omitted from $\mathcal{N}_{h}$ are called exceptional.

TheOrem 2.1. For any nearly convenient series $h \in \mathbf{C}\{X, Y\}$ with a singularity at $0 \in \mathbf{C}^{2}$ and with nonempty reduced Newton polygon $\mathcal{N}_{h}^{*}$ we have

$$
l_{0}(\operatorname{grad} h) \geq \max _{S \in \mathcal{N}_{h}^{*}}\{\alpha(S), \beta(S)\}-1 .
$$

Moreover, if the series is non-degenerate in Kouchnirenko's sense, then the equality holds.

In connection with Theorem 2.1 let us notice that if the series $h$ is not nearly convenient, then $l_{0}(\operatorname{grad} h)=+\infty$. If $h$ is nearly convenient with a singularity at $0 \in \mathbf{C}^{2}$, but $\mathcal{N}_{h}^{*}=\emptyset$, then $l_{0}(\operatorname{grad} h)=1$. The assumption of Kouchnirenko's non-degeneracy is, in fact, equivalent to the assumption that the series is non-degenerate on each segment of the reduced Newton polygon $\mathcal{N}_{h}^{*}$. The reason is that any series is always non-degenerate on the exceptional segments. The idea of omission of the extreme segments of the Newton Polygon was used by Kucharz [Kuch2] in a different context.

Let $F^{*}$ be the segment of $\mathcal{N}_{h}^{*}$ nearest to the vertical axis and let $L^{*}$ be the last one nearest to the horizontal axis. To compute the number on the right-hand side of the formula given in the theorem it is useful to notice that

$$
\max _{S \in \mathcal{N}_{h}^{*}}\{\alpha(S), \beta(S)\}=\max \left\{\alpha\left(L^{*}\right), \beta\left(F^{*}\right)\right\} .
$$

EXAMPLE. Let us consider the series $h=X Y^{6}+X^{2} Y^{4}+X^{7} Y+X^{11}$. The series $h$ is non-degenerate, nearly convenient and the polygon $\mathcal{N}_{h}^{*}$ is nonempty (notice that the last segment of $\mathcal{N}_{h}$ is exceptional). It is easy to compute that $\alpha\left(L^{*}\right)=8 \frac{2}{3}$ and $\beta\left(F^{*}\right)=8$. From Theorem 2.1 we have $l_{0}(\operatorname{grad} h)=\max \left\{8 \frac{2}{3}, 8\right\}-1=7 \frac{2}{3}$ and so $\nu_{h}=\left[7 \frac{2}{3}\right]+1=8$. For the comparison, notice that from the Lichtin result we have $\nu_{\tilde{h}} \leq 12$ for almost all 
series $\tilde{h}$ with the same Newton diagram, and from the Fukui result we have $l_{0}(\operatorname{grad} h) \leq 10$ and $\nu_{h} \leq 11$

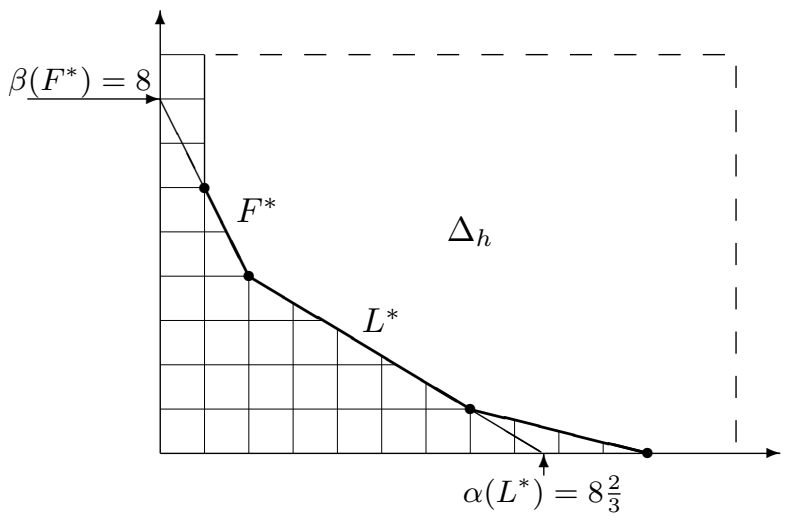

EXAMPLE. Let us consider the series $h=(X+Y)^{2}(X-Y)^{2}(2 X+4 Y)+X^{100}$. This series does not satisfy the non-degeneracy criterion, because the initial form $\operatorname{in}(h, S)$, corresponding to the unique segment $S$ of the Newton polygon, contains multiple factors. After linear variables transformation $X_{1}=X+Y$ and $Y_{1}=X-Y$ we obtain the nondegenerate series $\tilde{h}=X_{1}^{2} Y_{1}^{2}\left(3 X_{1}-Y_{1}\right)+\left(\frac{X_{1}+Y_{1}}{2}\right)^{100}$ with the same value of the Łojasiewicz exponent of the gradient. It is easy to notice that the vertices of the Newton diagram are the points: $(0,100),(2,3),(3,2),(100,0)$. None of the segments of the Newton polygon is exceptional. We have $\alpha\left(L^{*}\right)=\beta\left(F^{*}\right)=100$. Thus from Theorem 2.1 it follows that $l_{0}(\operatorname{grad} h)=l_{0}(\operatorname{grad} \tilde{h})=99$. In this case the Fukui result gives $l_{0}(\operatorname{grad} \tilde{h}) \leq 99$.

3. Newton diagrams and Puiseux series. In this section we collect elementary facts concerning Newton diagram and Puiseux series which will be useful in the proofs. Let $S$ be a segment of the Newton polygon of any series. Obviously, the line determined by $S$ has a negative slope. By the declivity of $S$ we mean the negative of the reciprocal of this slope. It is clear that any segment of the Newton polygon of any series has a positive declivity. Denote by $S_{1}$ and $S_{2}$ the projections of $S$ on the horizontal and vertical axes, respectively, and by $\left|S_{1}\right|,\left|S_{2}\right|$ their lengths. The declivity of $S$ is equal to $\frac{\left|S_{1}\right|}{\left|S_{2}\right|}$. The set of all segments of the Newton polygon of any series can be ordered according to the increasing declivity. Notice, that the "first" segment (of the minimal declivity) of any Newton polygon is nearest to the vertical axis, and the "last" one (of the maximal declivity) is nearest to the horizontal axis. The line passing through $(\alpha, \beta) \in \mathbf{R}^{2}$, parallel to $S$, intersects the horizontal axis at the point with the abscissa $\alpha+\frac{\left|S_{1}\right|}{\left|S_{2}\right|} \beta$, and the vertical axis - at the point with the ordinate $\alpha \frac{\left|S_{2}\right|}{\left|S_{1}\right|}+\beta$. If $(\alpha, \beta) \in S$, then $\alpha+\frac{\left|S_{1}\right|}{\left|S_{2}\right|} \beta=\alpha(S)$ and $\alpha \frac{\left|S_{2}\right|}{\left|S_{1}\right|}+\beta=\beta(S)$, where $\alpha(S)$ and $\beta(S)$ were defined in the previous section.

Let $h$ be a non-zero series. If $S$ is any segment of the Newton polygon of a series (not necessary from $\mathcal{N}_{h}$ ), then we put

$$
\alpha\left(S, \Delta_{h}\right)=\inf \left\{\alpha+\frac{\left|S_{1}\right|}{\left|S_{2}\right|} \beta:(\alpha, \beta) \in \operatorname{supp} h\right\} .
$$


and

$$
\beta\left(S, \Delta_{h}\right)=\inf \left\{\alpha \frac{\left|S_{2}\right|}{\left|S_{1}\right|}+\beta:(\alpha, \beta) \in \operatorname{supp} h\right\} .
$$

We have $\left|S_{1}\right| \beta\left(S, \Delta_{h}\right)=\left|S_{2}\right| \alpha\left(S, \Delta_{h}\right)$. Obviously, $\alpha\left(S, \Delta_{h}\right)=\alpha(S)$ and $\beta\left(S, \Delta_{h}\right)=\beta(S)$ for any $S \in \mathcal{N}_{h}$.

The numbers defined above have a simple geometrical meaning. The number $\alpha\left(S, \Delta_{h}\right)$ is equal to the abscissa of the point where the line supporting the diagram $\Delta_{h}$, parallel to the segment $S$, is crossed by the horizontal axis. Analogously, $\beta\left(S, \Delta_{h}\right)$ is equal to the ordinate of the point where the same line is crossed by the vertical axis.

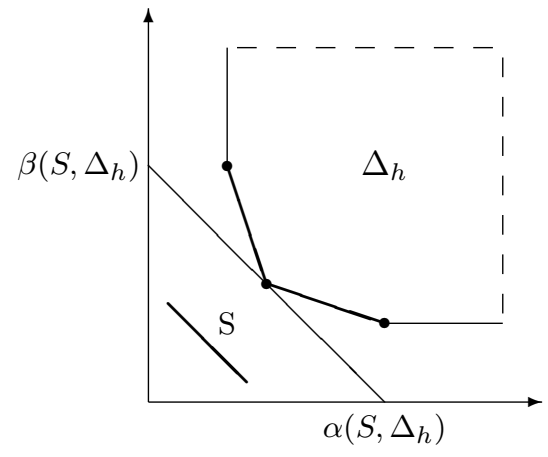

A series $h$ will be referred to as $X$-convenient if ord $h(X, 0)<+\infty$. It means that the Newton diagram $\Delta_{h}$ touches the horizontal axis, and the point $(\operatorname{ord} h(X, 0), 0)$ is the vertex of the diagram. If a series is not $X$-convenient $(h(X, 0)=0 \Leftrightarrow \operatorname{ord} h(X, 0)=+\infty)$, then its diagram does not touch the horizontal axis. We can analogously define the $Y$-conveniency of a series. A series, which is $X$ - and $Y$-convenient simultaneously, is called convenient. The conveniency of a series $h$ is equivalent to the condition $h(X, 0) h(0, Y) \neq 0$. Any nearly convenient series may be expressed in one of the four forms $\tilde{h}, X \tilde{h}, Y \tilde{h}, X Y \tilde{h}$, where $\tilde{h}$ is a convenient series. The following property gives a motivation for introducing the notion of nearly-conveniency.

Property 3.1. If a series $h \in \mathbf{C}\{X, Y\}$ has an isolated singularity at $0 \in \mathbf{C}^{2}$, then it is nearly convenient.

Proof. If $h$ is not nearly convenient, then it can be written in one of the two forms $h=X^{2} \tilde{h}$ or $h=Y^{2} \tilde{h}$, so the series has a multiple factor and therefore it cannot possess an isolated singularity.

Obviously, the opposite implication is not true. For example the series $h=(X-Y)^{2}$ is nearly convenient (even convenient) but it does not possess an isolated singularity. However, as a simple consequence of Theorem 2.1 we have

Property 3.2. If any nearly convenient series $h \in \mathbf{C}\{X, Y\}$ has a singularity at zero and is non-degenerate in Kouchnirenko's sense, then the singularity is isolated.

A ring (algebra) of the Puiseux series is the set $\mathbf{C}\{X\}^{*}=\bigcup_{k \geq 1} \mathbf{C}\left\{X^{\frac{1}{k}}\right\}$ with the naturally defined algebraic operations. For any non-zero Puiseux series $a(X)=a_{\theta} X^{\theta}+$ $a_{\theta^{\prime}} X^{\theta^{\prime}}+\ldots\left(\theta<\theta^{\prime}<\ldots\right.$ are positive rationals, $\left.a_{\theta}, a_{\theta^{\prime}}, \ldots \in \mathbf{C} \backslash\{0\}\right)$ we define ord $a(X)=\theta$ and in $a(X)=a_{\theta} X^{\theta}$. We put ord $0=+\infty$ and in $0=0$. Let $h(X, Y) \in$ 
$\mathbf{C}\{X, Y\}$. The solution of the equation $h(X, Y)=0$ in $\mathbf{C}\{X\}^{*}$ is the Puiseux series $a(X)$ such that $a(0)=0$ and $h(X, a(X))=0$. The smallest integer $m$ such that $\frac{\partial^{m} h}{\partial Y^{m}}(X, a(X)) \neq 0$ in $\mathbf{C}\{X\}^{*}$ is called the multiplicity of the solution $a(X)$ and will be denoted by $\kappa(a)$.

We have the following classical result concerning connections between the solutions of the equation $h(X, Y)=0$ and the Newton polygon $\mathcal{N}_{h}$.

Theorem 3.3 (Newton-Puiseux, see [BK], [Pł2]). Let $h(X, Y) \in \mathbf{C}\{X, Y\}$ be a nonzero series. Then, for any non-zero solution $a(X) \in \mathbf{C}\{X\}^{*}$ of the equation $h(X, Y)=0$ there exists a segment $S \in \mathcal{N}_{h}$, corresponding to the solution, such that ord $a(X)=\frac{\left|S_{1}\right|}{\left|S_{2}\right|}$ and $\operatorname{in}(h, S)(X$, in $a(X))=0$ in $\mathbf{C}\{X\}^{*}$. Moreover, for any segment $S \in \mathcal{N}_{h}$ there exist exactly $\left|S_{2}\right|$ solutions (counted with multiplicities) corresponding to $S$.

4. The Łojasiewicz exponent of a pair of series. In this section we consider a pair of non-zero series $H=(f, g), f(0,0)=g(0,0)=0$. This pair will be identified with the germ of holomorphic mapping $\left(\mathbf{C}^{2}, 0\right) \rightarrow\left(\mathbf{C}^{2}, 0\right)$. Our purpose is to give the best possible estimation of the value of exponent $l_{0}(f, g)$ using information from the diagrams $\Delta_{f}$ and $\Delta_{g}$. Under the assumption of non-degeneracy the exact value of the exponent will be obtained. The result is used in the proof of the main Theorem 2.1 in Section 6 . We use a standard definition of non-degeneracy of the pair $H=(f, g)$.

Definition 4.1. We say that a pair $(f, g)$ is non-degenerate if for any segment $S \in \mathcal{N}_{f}$ and any segment $T \in \mathcal{N}_{g}$ one of the following conditions is satisfied

(a) the segments $S$ and $T$ are not parallel,

(b) the segments $S$ and $T$ are parallel and the system $\operatorname{in}(f, S)=\operatorname{in}(g, T)=0$ has no solutions in $(\mathbf{C} \backslash\{0\}) \times(\mathbf{C} \backslash\{0\})$.

Notice that the non-degeneracy condition holds if one of the polygons $\mathcal{N}_{f}$ or $\mathcal{N}_{g}$ is empty.

Denote ord $H(X, 0)=\min \{\operatorname{ord} f(X, 0)$, ord $g(X, 0)\}$. The following theorem is the main result of this section.

ThEOREM 4.2. For any pair $H=(f, g)$ of non-zero series without constant term the Eojasiewicz exponent $l_{0}(H)$ is greater than or equal to the maximum of the six quantities

$$
\begin{aligned}
& \operatorname{ord} H(X, 0), \sup _{S \in \mathcal{N}_{f}} \alpha\left(S, \Delta_{g}\right), \sup _{T \in \mathcal{N}_{g}} \alpha\left(T, \Delta_{f}\right), \\
& \operatorname{ord} H(0, Y), \sup _{S \in \mathcal{N}_{f}} \beta\left(S, \Delta_{g}\right), \sup _{T \in \mathcal{N}_{g}} \beta\left(T, \Delta_{f}\right) .
\end{aligned}
$$

If the pair is non-degenerate then the equality holds.

The theorem will be proved later in this section. Now, we make some observations concerning calculation of the quantities appearing in the theorem. Next we give an example. First of all, let us notice that if the polygon $\mathcal{N}_{f}$ or $\mathcal{N}_{g}$ is empty, then the corresponding bound is equal to $-\infty$. If the polygon is nonempty, then the corresponding bound is equal to the maximum of a finite set of numbers. Directly from the definition of $\alpha\left(S, \Delta_{g}\right)$ and $\beta\left(S, \Delta_{g}\right)$ we obtain that if any segment $S$ is followed by any segment $S^{\prime}\left(\frac{\left|S_{1}\right|}{\left|S_{2}\right|} \leq \frac{\left|S_{1}^{\prime}\right|}{\left|S_{2}^{\prime}\right|}\right)$, 
then $\alpha\left(S, \Delta_{g}\right) \leq \alpha\left(S^{\prime}, \Delta_{g}\right)$ and $\beta\left(S, \Delta_{g}\right) \geq \beta\left(S^{\prime}, \Delta_{g}\right)$. Thus for a nonempty polygon $\mathcal{N}_{f}$ we have

$$
\sup _{S \in \mathcal{N}_{f}} \alpha\left(S, \Delta_{g}\right)=\alpha\left(L_{f}, \Delta_{g}\right) \text { and } \sup _{S \in \mathcal{N}_{f}} \beta\left(S, \Delta_{g}\right)=\beta\left(F_{f}, \Delta_{g}\right),
$$

where $F_{f}$ is the first and $L_{f}$ is the last segment of the polygon $\mathcal{N}_{f}$. Analogously, for a nonempty polygon $\mathcal{N}_{g}$, we have

$$
\sup _{T \in \mathcal{N}_{g}} \alpha\left(T, \Delta_{f}\right)=\alpha\left(L_{g}, \Delta_{f}\right) \text { and } \sup _{T \in \mathcal{N}_{g}} \beta\left(T, \Delta_{f}\right)=\beta\left(F_{g}, \Delta_{f}\right) .
$$

EXAmple. Let $H=(f, g)$ be a pair of series, where $f=Y^{4}+X Y^{2}+X^{3} Y+X^{5}$ and $g=X Y^{4}+X^{2} Y^{3}+X^{3} Y^{2}+X^{6} Y+X^{9}$. Because no segment of $\mathcal{N}_{f}$ is parallel to any segment of $\mathcal{N}_{g}$, the pair $(f, g)$ is non-degenerate. Moreover, ord $H(X, 0)=\min \{5,9\}=5$, $\alpha\left(L_{f}, \Delta_{g}\right)=7, \alpha\left(L_{g}, \Delta_{f}\right)=5$, and ord $H(0, Y)=\min \{4,+\infty\}=4, \beta\left(F_{f}, \Delta_{g}\right)=6$, $\beta\left(F_{g}, \Delta_{f}\right)=3$. From Theorem $4.2, l_{0}(H)=\max \{5,7,5,4,6,3\}=7$.
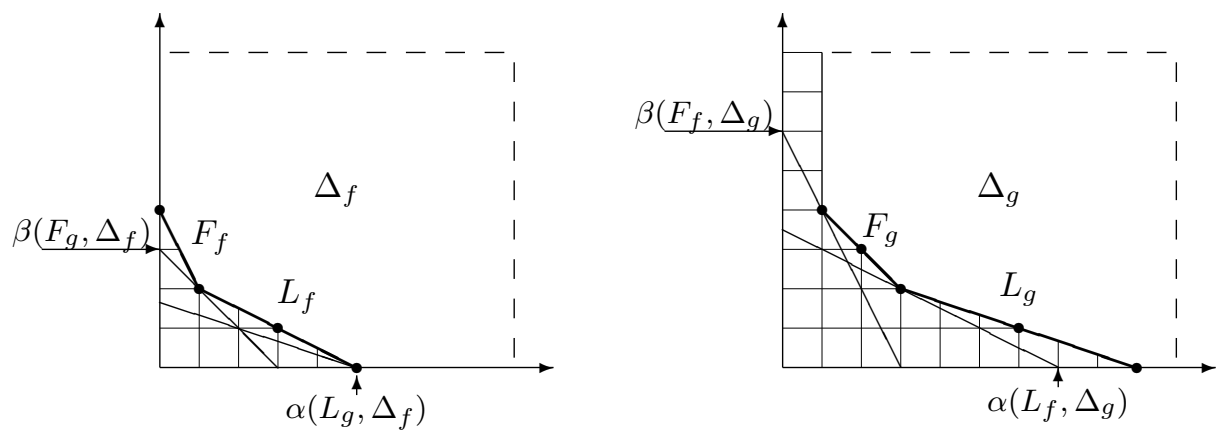

Definition 4.3. Let $H=(f, g)$ be a pair of the non-zero series without constant term. The relative Eojasiewicz exponent $l_{0}(H, X)$ of the pair $H$ with respect to the variable $X$ is the infimum of the set of all $\lambda>0$ such that

$$
\max \{|f(x, y)|,|g(x, y)|\} \geq c|x|^{\lambda},
$$

for $(x, y) \in \mathbf{C}^{2}$ in a neighbourhood of $0 \in \mathbf{C}^{2}$ and for a positive constant $c$.

We define $l_{0}(H, Y)$ in a similar way. The following lemma, which can easily be checked, enables us to reduce considerations to the relative exponents.

Lemma 4.4. $l_{0}(H)=\max \left\{l_{0}(H, X), l_{0}(H, Y)\right\}$.

In the sequel, we prove properties of $l_{0}(H, X)$. Corresponding properties of $l_{0}(H, Y)$ can be obtained by symmetry.

By an analytic arc we mean a pair of convergent series of one variable $p(T)=$ $\left(p_{1}(T), p_{2}(T)\right)$, such that $p_{1}(0)=p_{2}(0)=0$ and at least one of the pair components is non-zero series. By this conditions, the order of the arc, defined by the formula ord $p(T)=$ $\min \left\{\operatorname{ord} p_{1}(T)\right.$, ord $\left.p_{2}(T)\right\}$, is a positive integer. The following lemma can easily be proved.

Lemma 4.5. For any analytic arc $p(T)=\left(p_{1}(T), p_{2}(T)\right)$ such that $p_{1}(T) \neq 0$ in $\mathbf{C}\{T\}$, we have

$$
l_{0}(H, X) \geq \frac{\operatorname{ord} H(p(T))}{\operatorname{ord} p_{1}(T)} .
$$


Corollary. $l_{0}(H, X) \geq \operatorname{ord} H(X, 0)$.

Proof. We use the above lemma to $H$ and $p(T)=(T, 0)$.

The following theorem is an improved version of a result from [Pł2] (Proposition 3.2).

TheOREM 4.6. Let $H=(f, g)$ be a pair of non-zero series $f(X, Y), g(X, Y)$ in $\mathbf{C}\{X, Y\}$ without constant term. Let $\mathcal{A}$ be the set of all solutions of the equation $f(X, Y)=0$ in $\mathbf{C}\{X\}^{*}$ and let $\mathcal{B}$ be the set of all solutions of the equation $g(X, Y)=0$ in $\mathbf{C}\{X\}^{*}$. The case of lack of solution is admitted. Then

$$
l_{0}(H, X)=\max \left\{\operatorname{ord} H(X, 0), \sup _{a \in \mathcal{A}} \operatorname{ord} g(X, a(X)), \sup _{b \in \mathcal{B}} \operatorname{ord} f(X, b(X))\right\} .
$$

Proof. First, we prove that $l_{0}(H, X) \geq \operatorname{ord} g(X, a(X))$ for any solution $a(X) \in \mathcal{A}$ and $l_{0}(H, X) \geq \operatorname{ord} f(X, b(X))$ for any solution $b(X) \in \mathcal{B}$. By symmetry, it is sufficient to prove the first inequality. Fixing the solution $a(X)$ and choosing an integer $d \geq 1$ such that $a\left(T^{d}\right) \in \mathbf{C}\{T\}$ we consider the arc $p(T)=\left(T^{d}, a\left(T^{d}\right)\right)$. It is easy to notice that $H(p(T))=\left(0, g\left(T^{d}, a\left(T^{d}\right)\right)\right)$, so ord $H(p(T))=\operatorname{ord} g\left(T^{d}, a\left(T^{d}\right)\right)=d \operatorname{ord} g(X, a(X))$, and then, by Lemma 4.5,

$$
l_{0}(H, X) \geq \frac{\operatorname{ord} H(p(T))}{\operatorname{ord} T^{d}}=\operatorname{ord} g(X, a(X)) .
$$

Combining the above considerations with the corollary to Lemma 4.5 we end the proof of the lower estimation. In order to get the upper estimation we use the following lemma of the norm of a polynomial mapping ([Pł2], Lemma 3.1).

LEMma 4.7. If $w=(u, v)$ is a pair of non-zero polynomials $u(Y), v(Y) \in \mathbf{C}[Y]$, then for every $y \in \mathbf{C}$

$$
\max \{|u(y)|,|v(y)|\} \geq 2^{-n} \min \left\{|w(0)|, \inf _{\eta \in u^{-1}(0)}|v(\eta)|, \inf _{\eta \in v^{-1}(0)}|u(\eta)|\right\},
$$

where $n=\max \{\operatorname{deg} u, \operatorname{deg} v\}$.

Let $f(X, Y)=\tilde{f}(X, Y) \hat{f}(X, Y)$ and $g(X, Y)=\tilde{g}(X, Y) \hat{g}(X, Y)$ be the factorizations of the series into products of the invertible series $\tilde{f}(X, Y), \tilde{g}(X, Y)$ in the ring $\mathbf{C}\{X, Y\}$ and the distinguished polynomials $\hat{f}(X, Y), \hat{g}(X, Y) \in \mathbf{C}\{X\}[Y]$ such that

$$
\hat{f}(X, Y)=X^{r} \prod_{a \in \mathcal{A}}(Y-a(X))^{\kappa(a)} \text { and } \hat{g}(X, Y)=X^{s} \prod_{b \in \mathcal{B}}(Y-b(X))^{\kappa(b)},
$$

where $r=\min \{\alpha:(\alpha, \beta) \in \operatorname{supp} f\}$ and $s=\min \{\alpha:(\alpha, \beta) \in \operatorname{supp} g\}$. The existence of such factorizations comes from the Weierstrass preparation theorem and the NewtonPuiseux Theorem 3.3 (we use convention $\prod_{\emptyset}=1$ ). Let us choose an integer number $d \geq 1$ such that all the series $a\left(T^{d}\right)$ for $a \in \mathcal{A}$, and $b\left(T^{d}\right)$ for $b \in \mathcal{B}$ are in the ring $\mathbf{C}\{T\}$. If both equations have no non-zero solutions, then we put $d=1$. We fix neighbourhoods of zero $U_{1}, U_{2} \subset \mathbf{C}$ in such a way that for every $t \in U_{1}$ and for every $y \in U_{2}$ all the considered series are convergent and the estimates $\left|\tilde{f}\left(t^{d}, y\right)\right| \geq c,\left|\tilde{g}\left(t^{d}, y\right)\right| \geq c$ hold for a certain $c>0$. Hence

$$
\left|f\left(t^{d}, y\right)\right| \geq c\left|t^{d r} \prod_{a \in \mathcal{A}}\left(y-a\left(t^{d}\right)\right)^{\kappa(a)}\right| \text { and }\left|g\left(t^{d}, y\right)\right| \geq c\left|t^{d s} \prod_{b \in \mathcal{B}}\left(y-b\left(t^{d}\right)\right)^{\kappa(b)}\right|
$$

for $t \in U_{1}$ and $y \in U_{2}$. The formulae in modules, on the right-hand sides of the above inequalities, are polynomials with respect to $y$. Let $n$ be the maximum degree of these 
polynomials. Applying Lemma 4.7 to these polynomials we obtain

$$
\begin{aligned}
& \max \left\{\left|f\left(t^{d}, y\right)\right|,\left|g\left(t^{d}, y\right)\right|\right\} \geq \\
& \quad \geq c \cdot 2^{-n} \min \left\{\left|\hat{H}\left(t^{d}, 0\right)\right|, \inf _{a \in \mathcal{A}}\left|\hat{g}\left(t^{d}, a\left(t^{d}\right)\right)\right|, \inf _{b \in \mathcal{B}}\left|\hat{f}\left(t^{d}, b\left(t^{d}\right)\right)\right|\right\},
\end{aligned}
$$

where $\hat{H}=(\hat{f}, \hat{g})$. If at least one of the series $\hat{H}\left(T^{d}, 0\right), \hat{g}\left(T^{d}, a\left(T^{d}\right)\right), \hat{f}\left(T^{d}, b\left(T^{d}\right)\right)$ is the zero series, then the pair $H=(f, g)$ vanishes on an analytic arc, and then $l_{0}(H, X)=+\infty$. Also at least one of the orders on the right-hand side of the formula in the theorem is equal to $+\infty$. So, the theorem is proved for this case. Now, we may assume that each of the considered series is non-zero. Hence

$$
\begin{aligned}
& \max \left\{\left|f\left(t^{d}, y\right)\right|,\left|g\left(t^{d}, y\right)\right|\right\} \geq \\
& \geq c^{\prime}|t|^{\max \left\{\operatorname{ord} \hat{H}\left(T^{d}, 0\right), \sup _{a \in \mathcal{A}} \text { ord } \hat{g}\left(T^{d}, a\left(T^{d}\right)\right), \sup _{b \in \mathcal{B}} \text { ord } \hat{f}\left(T^{d}, b\left(T^{d}\right)\right)\right\}} \\
& =c^{\prime}\left|t^{d}\right| \max \left\{\operatorname{ord} \hat{H}(X, 0), \sup _{a \in \mathcal{A}} \text { ord } \hat{g}(X, a(X)), \sup _{b \in \mathcal{B}} \text { ord } \hat{f}(X, b(X))\right\}
\end{aligned}
$$

for $t$ near $0 \in \mathbf{C}$ and $y \in U_{2}$. We use here an elementary property that

$$
\min \left\{\left|p_{1}(t)\right|, \ldots,\left|p_{s}(t)\right|\right\} \geq c|t|^{\max \left\{\text { ord } p_{1}(T), \ldots, \text { ord } p_{s}(T)\right\}}
$$

for non-zero $p_{1}(T), \ldots, p_{s}(T) \in \mathbf{C}\{T\}$ and $t$ sufficiently close to $0 \in \mathbf{C}$. The observation that ord $\hat{H}(X, 0)=\operatorname{ord} H(X, 0)$, ord $\hat{g}(X, a(X))=\operatorname{ord} g(X, a(X))$ and ord $\hat{f}(X, b(X))=$ ord $f(X, b(X))$ ends the proof of the theorem.

The result below shows an important connection between the Łojasiewicz exponent $l_{0}(H, X)$ and the Newton diagrams $\Delta_{f}$ and $\Delta_{g}$.

TheOREM 4.8. For any pair $H=(f, g)$ of non-zero series $f(X, Y), g(X, Y)$ in $\mathbf{C}\{X, Y\}$ without constant term

$$
l_{0}(H, X) \geq \max \left\{\operatorname{ord} H(X, 0), \sup _{S \in \mathcal{N}_{f}} \alpha\left(S, \Delta_{g}\right), \sup _{T \in \mathcal{N}_{g}} \alpha\left(T, \Delta_{f}\right)\right\} .
$$

If the pair is non-degenerate, then the equality holds.

Pr o of. First, we show that if $a(X) \in \mathbf{C}\{X\}^{*}$ is a solution of the equation $f(X, Y)=0$ corresponding to the segment $S \in \mathcal{N}_{f}\left(\operatorname{ord} a(X)=\frac{\left|S_{1}\right|}{\left|S_{2}\right|}\right)$, then ord $g(X, a(X)) \geq \alpha\left(S, \Delta_{g}\right)$, and if additionally the pair $H=(f, g)$ is non-degenerate, then ord $g(X, a(X))=\alpha\left(S, \Delta_{g}\right)$. The Newton-Puiseux theorem gives

$$
\operatorname{in}(f, S)(X, \text { in } a(X))=0
$$

in $\mathbf{C}\{X\}^{*}$. Let $g(X, Y)=\sum g_{\alpha \beta} X^{\alpha} Y^{\beta}$. Then

$$
\begin{aligned}
\operatorname{ord} g(X, a(X)) & =\operatorname{ord}\left\{\sum_{(\alpha, \beta) \in \operatorname{supp} g} g_{\alpha \beta} X^{\alpha}\left(c X^{\frac{\left|S_{1}\right|}{\left|S_{2}\right|}}+\ldots\right)^{\beta}\right\} \\
& \geq \inf \left\{\alpha+\frac{\left|S_{1}\right|}{\left|S_{2}\right|} \beta:(\alpha, \beta) \in \operatorname{supp} g\right\}=\alpha\left(S, \Delta_{g}\right) .
\end{aligned}
$$

Let $Z \subset \operatorname{supp} g$ be the set of all $(\alpha, \beta) \in \operatorname{supp} g$ for which the linear form $(\alpha, \beta) \mapsto$ $\alpha+\frac{\left|S_{1}\right|}{\left|S_{2}\right|} \beta$ attains its minimum $\alpha\left(S, \Delta_{g}\right)$ on $\operatorname{supp} h$. The above considerations show that ord $g(X, a(X))>\alpha\left(S, \Delta_{g}\right)$ if and only if

$$
\sum_{(\alpha, \beta) \in Z} g_{\alpha \beta} X^{\alpha}(\operatorname{in} a(X))^{\beta}=0 .
$$


This situation is possible only if the set $Z$ has at least two elements (in order to obtain a reduction of the terms). In this case there exists the segment $T \in \mathcal{N}_{g}$ such that $\frac{\left|S_{1}\right|}{\left|S_{2}\right|}=\frac{\left|T_{1}\right|}{\left|T_{2}\right|}$ and $Z=T \cap \operatorname{supp} g$. Hence

$$
\sum_{(\alpha, \beta) \in Z} g_{\alpha \beta} X^{\alpha} Y^{\beta}=\operatorname{in}(g, T) .
$$

By (4) we have in $(g, T)(X$, in $a(X))=0$ in $\mathbf{C}\{X\}^{*}$, which in addition to (3) means that the system $\operatorname{in}(f, S)=\operatorname{in}(g, T)=0$ has a solution in $(\mathbf{C} \backslash\{0\}) \times(\mathbf{C} \backslash\{0\})$. Consequently, the pair $H=(f, g)$ is degenerate. So, we obtain ord $g(X, a(X))=\alpha\left(S, \Delta_{g}\right)$ for non-degenerate pairs. Analogously, we prove that for any solution $b(X) \in \mathbf{C}\{X\}^{*}$ of the equation $g(X, Y)=0$, corresponding to the segment $T \in \mathcal{N}_{g}\left(\operatorname{ord} b(X)=\frac{\left|T_{1}\right|}{\left|T_{2}\right|}\right)$, ord $g(X, b(X)) \geq \alpha\left(T, \Delta_{g}\right)$, with equality for non-degenerate pairs.

Zero solutions of the equations $f(X, Y)=0$ and $g(X, Y)=0$ may also exist. If $a(X)=0$ is a solution of $f(X, Y)=0$, then $\operatorname{ord} g(X, a(X))=\operatorname{ord} g(X, 0)=\operatorname{ord} H(X, 0)$, and similarly, if $b(X)=0$ is a solution of $g(X, Y)=0$, then ord $f(X, b(X))=$ ord $f(X, 0)=$ ord $H(X, 0)$. From Theorem 4.6 we obtain

$$
\begin{aligned}
l_{0}(H, X) & =\max \left\{\operatorname{ord} H(X, 0), \sup _{a \in \mathcal{A}} \operatorname{ord} g(X, a(X)), \sup _{b \in \mathcal{B}} \operatorname{ord} f(X, b(X))\right\} \\
& \geq \max \left\{\operatorname{ord} H(X, 0), \sup _{S \in \mathcal{N}_{f}} \alpha\left(S, \Delta_{g}\right), \sup _{T \in \mathcal{N}_{g}} \alpha\left(T, \Delta_{f}\right),\right\},
\end{aligned}
$$

with the equality for non-degenerate pairs.

From the theorem proved above, by symmetry, we have

THEOREM 4.9. For any pair $H=(f, g)$ of non-zero series without constant term $f(X, Y), g(X, Y) \in \mathbf{C}\{X, Y\}$

$$
l_{0}(H, Y) \geq \max \left\{\operatorname{ord} H(0, Y), \sup _{S \in \mathcal{N}_{f}} \beta\left(S, \Delta_{g}\right), \sup _{T \in \mathcal{N}_{g}} \beta\left(T, \Delta_{f}\right)\right\} .
$$

If the pair is non-degenerate, then the equality holds.

Now, Theorem 4.2 is a direct consequence of Lemma 4.4 and Theorems 4.8 and 4.9 .

5. The Newton diagram of series and diagrams of derivatives. In this section, our purpose is to compare the Newton polygons of the series $h, \frac{\partial h}{\partial X}$ and $\frac{\partial h}{\partial Y}$. We start from the comparison of the Newton polygons $\mathcal{N}_{h}$ and $\mathcal{N}_{\frac{\partial h}{\partial Y}}$.

It is useful to consider the vertices of the Newton diagram. The vertices can be ordered in such a way that the sequence of their abscissae is strongly increasing and the sequence of their ordinates is strongly decreasing. We say that a vertex of the $\Delta_{\frac{\partial h}{\partial Y}}$ is standard if it equals $(\mu, \nu-1)$ for a vertex $(\mu, \nu)$ of $\Delta_{h}$. We have an elementary fact that any vertex of $\Delta_{\frac{\partial h}{\partial Y}}$ which is followed by a standard one is also standard. A segment $T$ of $\mathcal{N}_{\frac{\partial h}{\partial Y}}$ is standard if its both ends are the standard vertices of $\Delta_{\frac{\partial h}{\partial Y}}$. Then, $T=S-(0,1)\left({ }^{2}\right)$ for some $S \in \mathcal{N}_{h}$ and obviously

$$
\operatorname{in}\left(\frac{\partial h}{\partial Y}, T\right)=\frac{\partial}{\partial Y} \operatorname{in}(h, S)
$$

$\left(^{2}\right)$ Symbol $S-(0,1)$ means the image of $S$ in the translation $(\alpha, \beta) \mapsto(\alpha, \beta-1)$. 
It is easy to notice that if $(\mu, \nu)$ is a vertex of $\Delta_{h}$ such that $\nu>0$, then $(\mu, \nu-1)$ is the standard vertex of $\Delta_{\frac{\partial h}{\partial Y}}$. We have

Property 5.1. If $(\mu, \nu)$ is the vertex of the diagram $\Delta_{h}$ with the minimal positive ordinate, then $(\mu, \nu-1)$ is the last standard vertex of $\Delta_{\frac{\partial h}{\partial Y}}$.

Proof. If $(\hat{\mu}, \hat{\nu})$ were the standard vertex of $\Delta_{\frac{\partial h}{\partial Y}}$ which lies below $(\mu, \nu-1)$, then $(\hat{\mu}, \hat{\nu}+1)$ would be the vertex of $\Delta_{h}$ with positive ordinate which lies below $(\mu, \nu)$. This is in the contradiction to the definition of the vertex $(\mu, \nu)$.

As an obvious consequence of the above property we have the following

COROllary 5.2. If $(\mu, \nu)$ is the vertex of the diagram $\Delta_{h}$ with the minimal positive ordinate, then a segment $T \in \mathcal{N}_{\frac{\partial h}{\partial Y}}$ is a standard segment if and only if $T$ lies over the vertex $(\mu, \nu-1)$ of the diagram $\Delta_{\frac{\partial h}{\partial Y}}$.
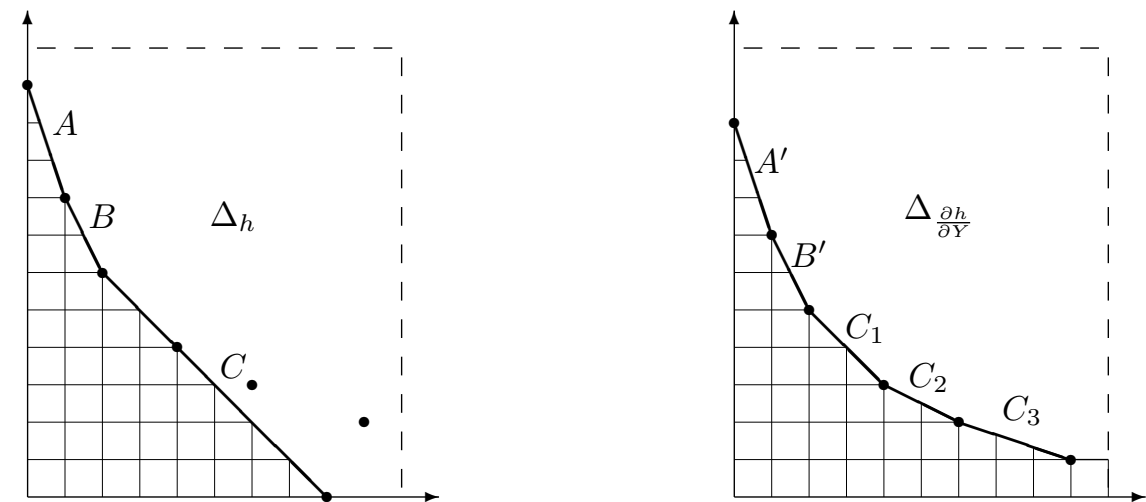

EXAMPLE. Let $h=Y^{11}+X Y^{8}+X^{2} Y^{6}+X^{4} Y^{4}+X^{6} Y^{3}+X^{9} Y^{2}+X^{8}$. The polygon $\mathcal{N}_{h}$ contains three segments $A, B, C$ which join the vertices $(0,11),(1,8),(2,6),(8,0)$. The polygon $\mathcal{N}_{\frac{\partial h}{\partial Y}}$ of the derivative $\frac{\partial h}{\partial Y}=11 Y^{10}+8 X Y^{7}+6 X^{2} Y^{5}+4 X^{4} Y^{3}+3 X^{6} Y^{2}+2 X^{9} Y$ contains five segments $A^{\prime}, B^{\prime}, C_{1}, C_{2}, C_{3}$ which join the vertices $(0,10),(1,7),(2,5)$, $(4,3),(6,2),(9,1)$. The vertex $(2,5)$ of the diagram $\Delta_{\frac{\partial h}{\partial Y}}$ separates the standard segments $A^{\prime}, B^{\prime}$ from the non-standard $C_{1}, C_{2}, C_{3}$. This vertex is the image in the translation $(\mu, \nu) \mapsto(\mu, \nu-1)$ of the vertex $(2,6)$ of the diagram $\Delta_{h}$ with the minimal positive ordinate. Let us notice that the non-standard segment $C_{1}$ is parallel to the last segment $C$ of the polygon $\mathcal{N}_{h}$.
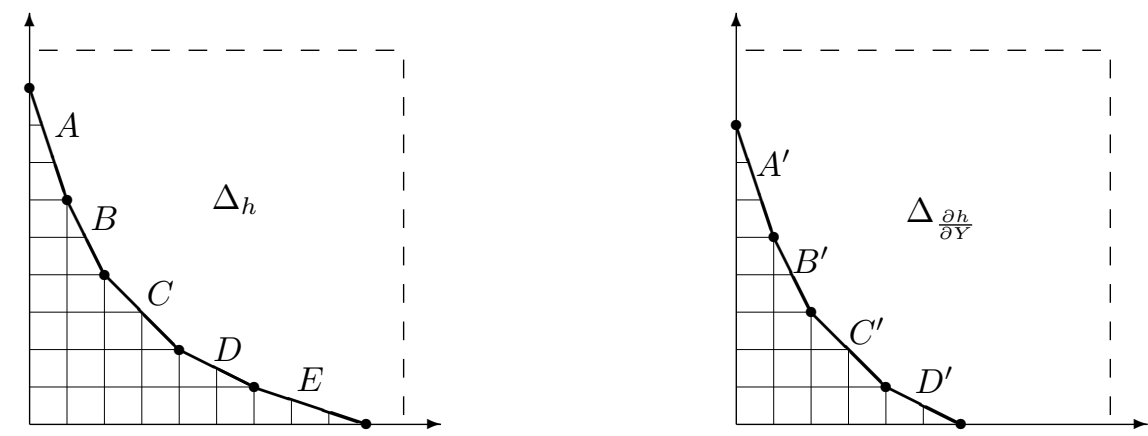
EXAMPLE. Let $h=Y^{9}+X Y^{6}+X^{2} Y^{4}+X^{4} Y^{2}+X^{6} Y+X^{9}$. The polygon $\mathcal{N}_{h}$ consists of five segments $A, B, C, D, E$ which join the vertices $(0,9),(1,6),(2,4),(4,2),(6,1)$, $(9,0)$. The polygon $\mathcal{N}_{\frac{\partial h}{\partial Y}}$ of the derivative $\frac{\partial h}{\partial Y}=9 Y^{8}+6 X Y^{5}+4 X^{2} Y^{3}+2 X^{4} Y+X^{6}$ consists of four segments $A^{\prime}, B^{\prime}, C^{\prime}, D^{\prime}$ which join the vertices $(0,8),(1,5),(2,3),(4,1)$, $(6,0)$. All the segments of the polygon $\mathcal{N}_{\frac{\partial h}{\partial Y}}$ are standard. There is no segment of the polygon $\mathcal{N}_{\frac{\partial h}{\partial Y}}$ which corresponds to the exceptional segment $E$ of the polygon $\mathcal{N}_{h}$. The vertex $(6,0)$ of the diagram $\Delta_{\frac{\partial h}{\partial Y}}$ separates the standard segments from the non-standard ones (there is a lack of the second group). This vertex is the image in the translation $(\mu, \nu) \mapsto(\mu, \nu-1)$ of the vertex $(6,1)$ of the diagram $\Delta_{h}$, with the minimal positive ordinate.

The theorem given below provides a description of the polygon $\mathcal{N}_{\frac{\partial h}{\partial Y}}$.

THEOREM 5.3. If $h(X, Y)$ is a nearly convenient series with a singularity at zero, then there exists a vertex $(\mu, \nu)$ of the diagram $\Delta_{h}$ with the minimal positive ordinate. Moreover, we have

(a) If $\nu=1$, then the polygon $\mathcal{N}_{\frac{\partial h}{\partial Y}}$ contains only the standard segments.

(b) If $\nu>1$, then there exists a segment $L \in \mathcal{N}_{h}$ that joins the vertex $(\mu, \nu)$ with a vertex on the horizontal axis, and for any $T \in \mathcal{N}_{\frac{\partial h}{\partial Y}}$ exactly one of the following conditions is satisfied:

(i) $T$ is a standard segment,

(ii) $\frac{\left|L_{1}\right|}{\left|L_{2}\right|}<\frac{\left|T_{1}\right|}{\left|T_{2}\right|}$

(iii) $T$ is parallel to $L$ and

$$
\text { in }\left(\frac{\partial h}{\partial Y}, T\right)=\frac{\partial}{\partial Y} \operatorname{in}(h, L) .
$$

Proof. By the assumption of nearly-conveniency $h$ is non-zero. Then, it has at least one vertex. Assume, to obtain a contradiction, that a vertex of a positive ordinate does not exist. In such a situation the unique vertex of $\Delta_{h}$ lies on the horizontal axis, that means $h=X^{\alpha} \tilde{h}$ and $\tilde{h}(0,0) \neq 0$. Since $h$ has a singularity at $0 \in \mathbf{C}^{2}$ we have $\alpha \geq 2$, which is in the contradiction to nearly-conveniency of $h$.

Let $(\mu, \nu)$ be the vertex of $\Delta_{h}$ with the minimal positive ordinate. From Corollary 5.2 all the segments of $\mathcal{N}_{\frac{\partial h}{\partial Y}}$ which lie over the vertex $(\mu, \nu-1)$ are standard. If $\nu=1$, then there is no segment of $\mathcal{N}_{\frac{\partial h}{\partial Y}}$ which lies below this vertex. This ends the proof of (a).

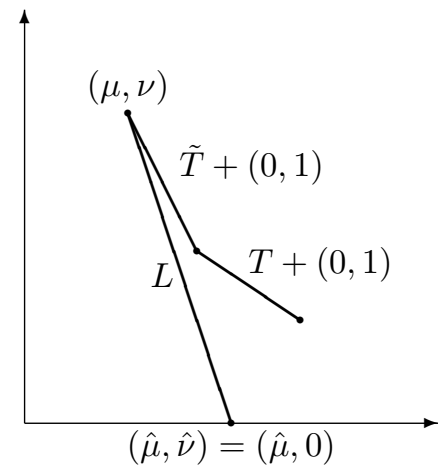


Now, assume that $\nu>1$. By nearly-conveniency, it is impossible for $(\mu, \nu)$ to be the last vertex of $\Delta_{h}$. Let $(\hat{\mu}, \hat{\nu})$ be the following vertex. We have $\hat{\nu}=0$, because in the opposite situation the vertex $(\mu, \nu)$ would not be the vertex of the minimal positive ordinate. Let $L$ be the segment of $\mathcal{N}_{h}$ that joins the vertices $(\mu, \nu)$ and $(\hat{\mu}, \hat{\nu})=(\hat{\mu}, 0)$. Obviously, it is the last segment of $\mathcal{N}_{h}$. Let $T \in \mathcal{N}_{\frac{\partial h}{\partial Y}}$. If $T$ lies over the vertex $(\mu, \nu-1)$, then it is standard, and condition (i) of part (b) is satisfied. If $T$ lies below the vertex $(\mu, \nu-1)$, then, obviously, there exists the segment $\tilde{T} \in \mathcal{N}_{\frac{\partial h}{\partial Y}}$ (followed by $T$ ) such that $(\mu, \nu-1)$ is its upper end.

The segment $\tilde{T}+(0,1)$ joins the vertex $(\mu, \nu)$ of $\Delta_{h}$ with a point from supp $h$. It is easy to derive in this case that the declivity of $\tilde{T}$ is greater than or equal to the declivity of $L$. Hence

$$
\frac{\left|L_{1}\right|}{\left|L_{2}\right|} \leq \frac{\left|\tilde{T}_{1}\right|}{\left|\tilde{T}_{2}\right|} \leq \frac{\left|T_{1}\right|}{\left|T_{2}\right|}
$$

If $\frac{\left|L_{1}\right|}{\left|L_{2}\right|}<\frac{\left|T_{1}\right|}{\left|T_{2}\right|}$, then the condition (ii) of (b) is satisfied. In the case $\frac{\left|L_{1}\right|}{\left|L_{2}\right|}=\frac{\left|T_{1}\right|}{\left|T_{2}\right|}$ we have

$$
\frac{\left|L_{1}\right|}{\left|L_{2}\right|}=\frac{\left|\tilde{T}_{1}\right|}{\left|\tilde{T}_{2}\right|}=\frac{\left|T_{1}\right|}{\left|T_{2}\right|}
$$

That means $T=\tilde{T}$ (two parallel segments of the same polygon coincide) and the segments $L$ and $T$ are parallel. Now, we have $T+(0,1) \subset L$ and

$$
\operatorname{in}(h, L)=\sum_{(\alpha, \beta) \in T+(0,1)} h_{\alpha \beta} X^{\alpha} Y^{\beta}+h_{\hat{\mu}, 0} X^{\hat{\mu}} .
$$

Differentiating the formula with respect to the variable $Y$ we obtain (5), which completes the proof of Theorem 5.3.

A simple consequence of the theorem just proved is

COROLlary 5.4. If $h(X, Y)$ is a nearly convenient series with a singularity at zero, then for any $T \in \mathcal{N}_{\frac{\partial h}{\partial Y}}$ exactly one of two conditions is satisfied:

(a) $T$ is parallel to a segment $S \in \mathcal{N}_{h}$ and then

$$
\text { in }\left(\frac{\partial h}{\partial Y}, T\right)=\frac{\partial}{\partial Y} \operatorname{in}(h, S) .
$$

(b) For every $S \in \mathcal{N}_{h} \frac{\left|S_{1}\right|}{\left|S_{2}\right|}<\frac{\left|T_{1}\right|}{\left|T_{2}\right|}$.

Proof. Let $T \in \mathcal{N}_{\frac{\partial h}{\partial Y}}$. If $T$ is parallel to a segment of $\mathcal{N}_{h}$, then it is standard or the condition described in the part (b)-(iii) of Theorem 5.3 is satisfied. Then (6) holds. If $T$ is not parallel to any segment of $\mathcal{N}_{h}$, then the situation described in the part (b)-(ii) of Theorem 5.3 is only possible. Since the segment $L$ (which joins the vertex $(\mu, \nu)$ with the vertex on the horizontal axis) is the last segment of $\mathcal{N}_{h}$, we have $\frac{\left|S_{1}\right|}{\left|S_{2}\right|} \leq \frac{\left|L_{1}\right|}{\left|L_{2}\right|}<\frac{\left|T_{1}\right|}{\left|T_{2}\right|}$ for every $S \in \mathcal{N}_{h}$.

The following Theorem 5.5 and Corollary 5.6 concern the description of the polygon $\mathcal{N}_{\frac{\partial h}{\partial X}}$. They can be obtained from Theorem 5.3 and Corollary 5.4, respectively, by changing the role of the variables $X$ and $Y$.

Analogously, as in the case of the Newton polygon of $\frac{\partial h}{\partial Y}$, we define a standard segment $R$ of $\mathcal{N}_{\frac{\partial h}{\partial Y}}$ to be of the form $S-(1,0)$ for any $S \in \mathcal{N}_{h}$. 
THEOREM 5.5. If $h(X, Y)$ is a nearly convenient series with a singularity at zero, then there exists the vertex $(\mu, \nu)$ of the diagram $\Delta_{h}$ with the minimal positive abscissa. Moreover, we have

(a) If $\mu=1$, then the polygon $\mathcal{N}_{\frac{\partial h}{\partial X}}$ contains only the standard segments.

(b) If $\mu>1$, then there exists a segment $F \in \mathcal{N}_{h}$ that joins a vertex on the axis with the vertex $(\mu, \nu)$, and for any $R \in \mathcal{N}_{\frac{\partial h}{\partial X}}$ exactly one of the following conditions is satisfied:

(i) $R$ is a standard segment,

(ii) $\frac{\left|R_{1}\right|}{\left|R_{2}\right|}<\frac{\left|F_{1}\right|}{\left|F_{2}\right|}$,

(iii) $R$ is parallel to the segment $F$ and then

$$
\text { in }\left(\frac{\partial h}{\partial X}, R\right)=\frac{\partial}{\partial X} \operatorname{in}(h, F) .
$$

COROLlary 5.6. If $h(X, Y)$ is a nearly convenient series with a singularity at zero, then for any $R \in \mathcal{N}_{\frac{\partial h}{\partial X}}$ exactly one of two conditions is satisfied

(a) $R$ is parallel to a segment $S \in \mathcal{N}_{h}$ and then

$$
\text { in }\left(\frac{\partial h}{\partial X}, R\right)=\frac{\partial}{\partial X} \operatorname{in}(h, S) .
$$

(b) For every $S \in \mathcal{N}_{h} \frac{\left|R_{1}\right|}{\left|R_{2}\right|}<\frac{\left|S_{1}\right|}{\left|S_{2}\right|}$.

A direct consequence of the results presented above is the following

THEOREM 5.7. Let $h=h(X, Y) \in \mathbf{C}\{X, Y\}$ be a nearly convenient series with a singularity at zero. If the series is non-degenerate in Kouchnirenko's sense, then the pair $\left(\frac{\partial h}{\partial X}, \frac{\partial h}{\partial Y}\right)$ is non-degenerate.

Proof. If one of the polygons $\mathcal{N}_{\frac{\partial h}{\partial X}}$ or $\mathcal{N}_{\frac{\partial h}{\partial Y}}$ is empty, then the non-degeneracy condition of the pair is trivially satisfied. Therefore, we can assume that both polygons are nonempty. Let $R \in \mathcal{N}_{\frac{\partial h}{\partial X}}$ and $T \in \mathcal{N}_{\frac{\partial h}{\partial Y}}$. If the segments $R$ and $T$ are not parallel, then they satisfy the non-degeneracy condition. Let us assume that they are parallel. In this case we have to show that the system of equations in $\left(\frac{\partial h}{\partial X}, R\right)=$ in $\left(\frac{\partial h}{\partial Y}, T\right)=0$ has no solutions in $(\mathbf{C} \backslash\{0\}) \times(\mathbf{C} \backslash\{0\})$. We assert, that there exists a segment of $\mathcal{N}_{h}$ parallel to $R$ and $T$. If it did not exist, then by Corollary $5.6 \frac{\left|R_{1}\right|}{\left|R_{2}\right|}<\frac{\left|S_{1}\right|}{\left|S_{2}\right|}$ for every $S \in \mathcal{N}_{h}$, and by Corollary $5.4 \frac{\left|S_{1}\right|}{\left|S_{2}\right|}<\frac{\left|T_{1}\right|}{\left|T_{2}\right|}$ for every $S \in \mathcal{N}_{h}$. Then $\frac{\left|R_{1}\right|}{\left|R_{2}\right|}<\frac{\left|T_{1}\right|}{\left|T_{2}\right|}$, which would be in the contradiction to the parallelism of $R$ and $T$. Hence, the segment $S \in \mathcal{N}_{h}$ parallel to $R$ and $T$ exists. Corollaries 5.6 and 5.4 give

$$
\text { in }\left(\frac{\partial h}{\partial X}, R\right)=\frac{\partial}{\partial X} \operatorname{in}(h, S) \text { and in }\left(\frac{\partial h}{\partial Y}, T\right)=\frac{\partial}{\partial Y} \operatorname{in}(h, S) .
$$

The non-degeneracy of the series $h$ on the segment $S$ means that the system $\frac{\partial}{\partial X} \operatorname{in}(h, S)=$ $\frac{\partial}{\partial Y} \operatorname{in}(h, S)=0$ has no solution in $(\mathbf{C} \backslash\{0\}) \times(\mathbf{C} \backslash\{0\})$. By the equalities proved above the system in $\left(\frac{\partial h}{\partial X}, R\right)=$ in $\left(\frac{\partial h}{\partial Y}, T\right)=0$ has no solution in $(\mathbf{C} \backslash\{0\}) \times(\mathbf{C} \backslash\{0\})$ either. This completes the proof of the theorem. 
6. Proof of the main theorem. Let $h \in \mathbf{C}\{X, Y\}$ be a nearly convenient series with a singularity at zero and with the nonempty reduced Newton Polygon $\mathcal{N}_{h}^{*}$. By these assumptions the components of the pair $\operatorname{grad} h=\left(\frac{\partial h}{\partial X}, \frac{\partial h}{\partial Y}\right)$ are non-zero series without constant term. Applying Theorem 4.2 to this pair we see that the exponent $l_{0}(\operatorname{grad} h)$ is greater than or equal to the maximum of the six quantities

$$
\begin{aligned}
& \operatorname{ord} \operatorname{grad} h(X, 0), \sup _{R \in \mathcal{N}_{\frac{\partial h}{\partial X}}} \alpha\left(R, \Delta_{\frac{\partial h}{\partial Y}}\right), \sup _{T \in \mathcal{N}_{\frac{\partial h}{\partial Y}}} \alpha\left(T, \Delta_{\frac{\partial h}{\partial X}}\right), \\
& \operatorname{ord} \operatorname{grad} h(0, Y), \sup _{R \in \mathcal{N}_{\frac{\partial h}{\partial X}}} \beta\left(R, \Delta_{\frac{\partial h}{\partial Y}}\right), \sup _{T \in \mathcal{N}_{\frac{\partial h}{\partial Y}}} \beta\left(T, \Delta_{\frac{\partial h}{\partial X}}\right) .
\end{aligned}
$$

Theorems 4.2 and 5.7 imply that if the series $h$ is non-degenerate in Kouchnirenko's sense, then the equality holds. Let us denote by $M_{X}$ the maximum of three quantities in (7) and by $M_{Y}$ the maximum of three quantities in (8). In order to finish the proof of the main theorem it is sufficient to show that

$$
\max \left\{M_{X}, M_{Y}\right\}=\max \left\{\alpha\left(L^{*}\right), \beta\left(F^{*}\right)\right\}-1,
$$

where $F^{*}$ is the first and $L^{*}$ is the last segment of $\mathcal{N}_{h}^{*}$. The equality may be easily derived from the following

LEMMA 6.1. If any nearly convenient series $h$ with a singularity at zero has the nonempty reduced Newton polygon $\mathcal{N}_{h}^{*}$, then

(a) $\alpha\left(L^{*}\right)-1 \leq M_{X} \leq \max \left\{\alpha\left(L^{*}\right)-1, \alpha\left(L^{*}\right)-\frac{\left|L_{1}^{*}\right|}{\left|L_{2}^{*}\right|}\right\}$,

(b) $\beta\left(F^{*}\right)-1 \leq M_{Y} \leq \max \left\{\beta\left(F^{*}\right)-1, \beta\left(F^{*}\right)-\frac{\left|F_{2}^{*}\right|}{\left|F_{1}^{*}\right|}\right\}$.

Before giving the proof of the lemma we show how it implies (9). The consequence of (a) and (b) is the estimate $M_{2} \leq \max \left\{M_{X}, M_{Y}\right\} \leq M_{4}$, where $M_{2}$ is the maximum of the two numbers $\alpha\left(L^{*}\right)-1, \beta\left(F^{*}\right)-1$ and $M_{4}$ is the maximum of the four numbers $\alpha\left(L^{*}\right)-1, \alpha\left(L^{*}\right)-\frac{\left|L_{1}^{*}\right|}{\left|L_{2}^{*}\right|}, \beta\left(F^{*}\right)-1, \beta\left(F^{*}\right)-\frac{\left|F_{2}^{*}\right|}{\left|F_{1}^{*}\right|}$. It is enough to show that $M_{2} \geq M_{4}$. Since the segment $F^{*}$ is followed by the segment $L^{*}$, the declivity of $F^{*}$ is less than or equal to the declivity of $L^{*}$. Now, we consider three cases.

If $\frac{\left|F_{1}^{*}\right|}{\left|F_{2}^{*}\right|} \leq 1 \leq \frac{\left|L_{1}^{*}\right|}{\left|L_{2}^{*}\right|}$, then the inequality $M_{2} \geq M_{4}$ is obvious. If $1 \leq \frac{\left|F_{1}^{*}\right|}{\left|F_{2}^{*}\right|} \leq \frac{\left|L_{1}^{*}\right|}{\left|L_{2}^{*}\right|}$, then $M_{4}=\max \left\{\alpha\left(L^{*}\right)-1, \beta\left(F^{*}\right)-\frac{\left|F_{2}^{*}\right|}{\left|F_{1}^{*}\right|}\right\}$. We show that this maximum is equal to $\alpha\left(L^{*}\right)-1$. Using the equality $\frac{\beta\left(F^{*}\right)}{\alpha\left(F^{*}\right)}=\frac{\left|F_{2}^{*}\right|}{\left|F_{1}^{*}\right|}$ and the obvious inequality $\alpha\left(F^{*}\right) \leq \alpha\left(L^{*}\right)$ we obtain

$$
\beta\left(F^{*}\right)-\frac{\left|F_{2}^{*}\right|}{\left|F_{1}^{*}\right|}=\alpha\left(F^{*}\right) \frac{\left|F_{2}^{*}\right|}{\left|F_{1}^{*}\right|}-\frac{\left|F_{2}^{*}\right|}{\left|F_{1}^{*}\right|}=\left[\alpha\left(F^{*}\right)-1\right] \frac{\left|F_{2}^{*}\right|}{\left|F_{1}^{*}\right|} \leq \alpha\left(L^{*}\right)-1,
$$

thus $M_{2} \geq M_{4}$. The case $\frac{\left|F_{1}^{*}\right|}{\left|F_{2}^{*}\right|} \leq \frac{\left|L_{1}^{*}\right|}{\left|L_{2}^{*}\right|} \leq 1$ can be verified analogously.

Proof of Lemma 6.1. By symmetry, it is enough to prove part (a) of the lemma. First, we verify that for any $S \in \mathcal{N}_{h}$

$$
\alpha\left(S, \Delta_{\frac{\partial h}{\partial X}}\right)=\alpha(S)-1 \quad \text { and } \quad \alpha\left(S, \Delta_{\frac{\partial h}{\partial Y}}\right)=\alpha(S)-\frac{\left|S_{1}\right|}{\left|S_{2}\right|} .
$$

Let us consider a linear form $\phi(\alpha, \beta)=\alpha+\frac{\left|S_{1}\right|}{\left|S_{2}\right|} \beta$ on the real plane. An obvious inclusion $\operatorname{supp} \frac{\partial h}{\partial X} \subset \operatorname{supp} h-(1,0)$ helps us to prove the first formula in (10). We have $\alpha\left(S, \Delta_{\frac{\partial h}{\partial X}}\right)=\inf \phi\left(\operatorname{supp} \frac{\partial h}{\partial X}\right) \geq \inf \phi(\operatorname{supp} h)+\phi(-1,0)=\alpha\left(S, \Delta_{h}\right)+\phi(-1,0)=\alpha(S)-1$. 
To obtain the opposite inequality, notice that the segment $S$ has at least one end $(\mu, \nu)$ of the positive abscissa. Hence $(\mu-1, \nu) \in \operatorname{supp} \frac{\partial h}{\partial X}$ and therefore

$$
\alpha\left(S, \Delta_{\frac{\partial h}{\partial X}}\right) \leq(\mu-1)+\frac{\left|S_{1}\right|}{\left|S_{2}\right|} \nu=\alpha(S)-1,
$$

which ends the proof of the first formula in (10). The proof of the second formula is similar.

Let $(\mu, \nu)$ be the vertex of the diagram $\Delta_{h}$ with the minimal positive ordinate. Let us consider the case $\nu=1$. Now, the vertex $(\mu, \nu)=(\mu, 1)$ is the lower end of the segment $L^{*}$ (if a segment of $\mathcal{N}_{h}$ that joins the vertex $(\mu, \nu)$ with the horizontal axis exists, then it is exceptional). Since $(\mu, 1) \in L^{*}$ we have $\alpha\left(L^{*}\right)=\mu+\frac{\left|L_{1}^{*}\right|}{\left|L_{2}^{*}\right|}$. In the case under consideration the series $h$ may be written in the form

$$
h(X, Y)=a_{0}(X)+Y a_{1}(X)+Y^{2} \tilde{h}(X, Y),
$$

where ord $a_{1}(X)=\mu$. By the equality $\frac{\partial h}{\partial Y}(X, 0)=a_{1}(X)$ we have ord $\frac{\partial h}{\partial Y}(X, 0)=\mu$. Hence, we obtain

$$
\operatorname{ord} \operatorname{grad} h(X, 0) \leq \operatorname{ord} \frac{\partial h}{\partial Y}(X, 0)=\mu=\alpha\left(L^{*}\right)-\frac{\left|L_{1}^{*}\right|}{\left|L_{2}^{*}\right|} .
$$

From $(\mu, 1) \in \operatorname{supp} h$ follows that $(\mu, 0) \in \operatorname{supp} \frac{\partial h}{\partial Y}$. Therefore, for any $R \in \mathcal{N}_{\frac{\partial h}{\partial X}}$ we have $\alpha\left(R, \Delta_{\frac{\partial h}{\partial Y}}\right) \leq \mu+\frac{\left|R_{1}\right|}{\left|R_{2}\right|} \cdot 0=\mu$, hence

$$
\sup _{R \in \mathcal{N}_{\frac{\partial h}{\partial X}}} \alpha\left(R, \Delta_{\frac{\partial h}{\partial Y}}\right) \leq \mu=\alpha\left(L^{*}\right)-\frac{\left|L_{1}^{*}\right|}{\left|L_{2}^{*}\right|} .
$$

The segment $L=L^{*}-(0,1)$ is the standard segment of the polygon $\mathcal{N}_{\frac{\partial h}{\partial Y}}$, parallel to $L^{*}$. Obviously, $L$ is the last segment of $\mathcal{N}_{\frac{\partial h}{\partial Y}}$. Thus for any $T \in \mathcal{N}_{\frac{\partial h}{\partial Y}}$ we have $\alpha\left(T, \Delta_{\frac{\partial h}{\partial X}}\right) \leq$ $\alpha\left(L, \Delta_{\frac{\partial h}{\partial X}}\right)$ and

$$
\sup _{T \in \mathcal{N}_{\frac{\partial h}{\partial Y}}} \alpha\left(T, \Delta_{\frac{\partial h}{\partial X}}\right)=\alpha\left(L, \Delta_{\frac{\partial h}{\partial X}}\right) .
$$

Since the segments $L$ and $L^{*}$ are parallel we have $\alpha\left(L, \Delta_{\frac{\partial h}{\partial X}}\right)=\alpha\left(L^{*}, \Delta_{\frac{\partial h}{\partial X}}\right)$. The second formula of (10) gives $\alpha\left(L^{*}, \Delta_{\frac{\partial h}{\partial X}}\right)=\alpha\left(L^{*}\right)-1$. Consequently

$$
\sup _{T \in \mathcal{N}_{\frac{\partial h}{\partial Y}}} \alpha\left(T, \Delta_{\frac{\partial h}{\partial X}}\right)=\alpha\left(L^{*}\right)-1
$$

Joining the above equality with (11) and (12) we obtain part (a) of Lemma 6.1 for the case $\nu=1$.

Now, assume that $\nu>1$. According to Theorem 5.3 there exists a segment $L \in \mathcal{N}_{h}$ that joins the vertex $(\mu, \nu)$ with the vertex $(\hat{\mu}, 0)$ on the horizontal axis. Since $h$ has a singularity at zero we have $\hat{\mu}>1$. Therefore $L$ is not exceptional and $L=L^{*}$. By $(\hat{\mu}, 0) \in L^{*}$ we obtain $\alpha\left(L^{*}\right)=\hat{\mu}+\frac{\left|L_{1}^{*}\right|}{\left|L_{2}^{*}\right|} \cdot 0=\hat{\mu}$. The series $h$ may be written in the form

$$
h(X, Y)=a_{0}(X)+Y \tilde{h}(X, Y),
$$

where ord $a_{0}=\hat{\mu}$. We have ord $\frac{\partial h}{\partial X}(X, 0)=\operatorname{ord} a_{0}^{\prime}(X)=\hat{\mu}-1=\alpha\left(L^{*}\right)-1$. Therefore

$$
\operatorname{ord} \operatorname{grad} h(X, 0) \leq \operatorname{ord} \frac{\partial h}{\partial X}(X, 0)=\alpha\left(L^{*}\right)-1 .
$$


In a way analogous to earlier considerations, from the fact $(\hat{\mu}-1,0) \in \operatorname{supp} \frac{\partial h}{\partial X}$, we obtain

$$
\sup _{T \in \mathcal{N}_{\frac{\partial h}{\partial Y}}} \alpha\left(T, \Delta_{\frac{\partial h}{\partial X}}\right) \leq \hat{\mu}-1=\alpha\left(L^{*}\right)-1 .
$$

By Corollary 5.6 the declivity of any segment $R \in \mathcal{N}_{\frac{\partial h}{\partial X}}$ is not greater than the maximal declivity of the segments from $\mathcal{N}_{h}$. Hence $\frac{\left|R_{1}\right|}{\left|R_{2}\right|} \leq \frac{\left|L_{1}^{*}\right|}{\left|L_{2}^{*}\right|}$ for any $R \in \mathcal{N}_{\frac{\partial h}{\partial X}}$ and therefore

$$
\sup _{R \in \mathcal{N}_{\frac{\partial h}{\partial X}}} \alpha\left(R, \Delta_{\frac{\partial h}{\partial Y}}\right) \leq \alpha\left(L^{*}, \Delta_{\frac{\partial h}{\partial Y}}\right) .
$$

According to the second formula of (10) we have

$$
\sup _{R \in \mathcal{N}_{\frac{\partial h}{\partial X}}} \alpha\left(R, \Delta_{\frac{\partial h}{\partial Y}}\right) \leq \alpha\left(L^{*}\right)-\frac{\left|L_{1}^{*}\right|}{\left|L_{2}^{*}\right|} .
$$

Joining the last inequality with the estimates (13) and (14) we obtain

$$
M_{X} \leq \max \left\{\alpha\left(L^{*}\right)-1, \alpha\left(L^{*}\right)-\frac{\left|L_{1}^{*}\right|}{\left|L_{2}^{*}\right|}\right\} .
$$

In order to finish the proof of Lemma 6.1 it is sufficient to prove that $M_{X} \geq \alpha\left(L^{*}\right)-1$. We show that always at least one of the quantities from the definition of $M_{X}$ is greater than or equal to $\alpha\left(L^{*}\right)-1$.

First, assume that the polygon $\mathcal{N}_{\frac{\partial h}{\partial Y}}$ has non-standard segments. For any non-standard segment $T \in \mathcal{N}_{\frac{\partial h}{\partial Y}}$, by Corollary 5.4 we have $\frac{\left|L_{1}^{*}\right|}{\left|L_{2}^{*}\right|} \leq \frac{\left|T_{1}\right|}{\left|T_{2}\right|}$. Hence

$$
\alpha\left(L^{*}\right)-1=\alpha\left(L^{*}, \Delta_{\frac{\partial h}{\partial X}}\right) \leq \alpha\left(T, \Delta_{\frac{\partial h}{\partial X}}\right) \leq \sup _{T \in \mathcal{N}_{\frac{\partial h}{\partial Y}}} \alpha\left(T, \Delta_{\frac{\partial h}{\partial X}}\right) .
$$

Now, assume that non-standard segments of $\mathcal{N}_{\frac{\partial h}{\partial Y}}$ do not exist. By Theorem 5.3 nonstandard segments may only exist below the vertex $(\mu, \nu-1)$ of the diagram $\Delta_{\frac{\partial h}{\partial Y}}$. The lack of such segments means that the vertex $(\mu, \nu-1)$ is the last one. Then, there are no points of $\operatorname{supp} \frac{\partial h}{\partial Y}$ below the vertex $(\mu, \nu-1)$. Therefore

$$
\frac{\partial h}{\partial Y}=Y^{\nu-1} \hat{h}(X, Y)
$$

which, according to $\nu>1$, gives $\frac{\partial h}{\partial Y}(X, 0)=0$, and thus ord $\frac{\partial h}{\partial Y}(X, 0)=+\infty$. Finally, we obtain the equality

$$
\begin{aligned}
\operatorname{ord} \operatorname{grad} h(X, 0) & =\min \left\{\text { ord } \frac{\partial h}{\partial X}(X, 0), \text { ord } \frac{\partial h}{\partial Y}(X, 0)\right\}= \\
& =\min \left\{\alpha\left(L^{*}\right)-1,+\infty\right\}=\alpha\left(L^{*}\right)-1,
\end{aligned}
$$

which completes the proof of Lemma 6.1 and also the proof of the main theorem.

\section{References}

[BŁ] J. Bochnak and S. Łojasiewicz, A converse of the Kuiper-Kuo theorem, in: Proceedings of Liverpool Singularities-Symposium I, Lecture Notes in Math. 192, Springer, Berlin, 1971, 254-261.

[BK] E. Brieskorn and H. Knörer, Ebene algebraische Kurven, Birkhäuser, Basel, 1981. 
[ChK1] J. Chạdzyński and T. Krasiński, The Eojasiewicz exponent of an analytic mapping of two complex variables at an isolated zero, in: Singularities, Banach Center Publ. 20, PWN-Polish Science Publishers, Warszawa, 1988, 139-146.

[ChK2] J. Chạdzyński and T. Krasiński, Resultant and the Eojasiewicz exponent, Ann. Polon. Math. 61 (1995), 95-100.

[Fu] T. Fukui, Eojasiewicz type inequalities and Newton diagrams, Proc. Amer. Math. Soc. 112 (1991), 1169-1183.

[Kou] A. G. Kouchnirenko, Polyèdres de Newton et nombres de Milnor, Invent. Math. 32 (1976), 1-31.

[Kuch1] W. Kucharz, Examples in the theory of sufficiency of jets, Proc. Amer. Math. Soc. 96 (1986), 163-166.

[Kuch2] W. Kucharz, Newton polygons and topological determinancy of analytic germs, Period. Math. Hungar. 22 (1991), 129-132.

[Kuip] N. H. Kuiper, $C^{1}$-equivalence of functions near isolated critical points, in: Symposium on Infinite-Dimensional Topology, R. D. Anderson (ed.), Ann. of Math. Studies 69, Princeton Univ. Press, Princeton, 1972, 199-218.

[K] T. C. Kuo, On $C^{0}$ sufficiency of jets of potential functions, Topology 8 (1969), 167-171.

[KL] T. C. Kuo and Y. C. Lu, On analytic function germ of two complex variables, Topology 16 (1977), 299-310.

[LJT] M. Lejeune-Jalabert and B. Teissier, Cloture integral des idéaux et equisingularité, Centre Math. École Polytechnique, Paris, 1974.

[Li] B. Lichtin, Estimation of Eojasiewicz exponent and Newton polygons, Invent. Math. 64 (1981), 417-429.

[LCh] Y. C. Lu and S. S. Chang, On $C^{0}$ sufficiency of complex jets, Canad. J. Math. 25 (1973), 874-880.

[Ł] S. Łojasiewicz, Ensembles semi-analytiques, Inst. de Hautes Études Scientifiques, Bures-sur-Yvette, 1965.

[Pł1] A. Płoski, Une évaluation pour les sous-ensembles analytiques complexes, Bull. Polish Acad. Sci. Math. 31 (1983), 259-262.

[Pł2] A. Płoski, Newton polygons and the Eojasiewicz exponent of a holomorphic mapping of $\mathbf{C}^{2}$, Ann. Polon. Math. 51 (1990), 275-281.

[Te] B. Teissier, Variétés polaires, Invent. Math. 40 (1977), 267-292. 